\title{
Chromatin condensation retains the osteogenic transcription factor, RUNX2, in the nucleus of human mesenchymal stem cells
}

\author{
Masashi YAMAZAKI*, Hiromichi FUJIE* and Hiromi MIYOSHI* \\ *Department of Mechanical Systems Engineering, Graduate School of Systems Design, Tokyo Metropolitan University \\ 1-1 Minami-Osawa, Hachioji, Tokyo, Japan 192-0397 \\ E-mail: hiromi-miyoshi@tmu.ac.jp
}

Received: 19 February 2020; Revised: 20 April 2020; Accepted: 21 May 2020

\begin{abstract}
Mechanical forces transduced to the nucleus that affect intranuclear organization are critical regulators for directing the differentiation of mesenchymal stem cells (MSCs). The supraphysiological dose of a mechanical cue, which is from the stiff surface of the substrate like standard tissue culture polystyrene, biases the MSCs toward osteogenic differentiation with irreversible nuclear retention of an osteogenic master transcription factor, RUNX2. To study the mechanism underlying the loss of lineage plasticity and robustness of the irreversible osteogenic differentiation in human MSCs due to the mechanotransduction, we investigated how RUNX2 was retained in the nucleus focusing on the dynamic shuttling of a mechanotransducer, yes-associated protein (YAP), intranuclear chromatin organization, and their regulation by the actin cytoskeleton using pharmaceutical inhibitors to perturb the mechanical properties of chromatin and actin cytoskeleton. YAP and RUNX2 were localized in the nucleus of MSCs during the expansion culture on the polystyrene surfaces followed by 24 hours culture on the glass surface, at the time point just before the inhibitor treatments. The nuclear RUNX2 was diffused into the cytoplasm by chromatin decondensation through the inhibition of histone deacetylase. In contrast, RUNX2 localization was unaffected from the perturbations to the actin organization through the inhibition of actin polymerization and that of Rho kinase activity under the condition that chromatin condensation was maintained, although YAP was released from the nucleus to the cytoplasm. These findings suggest that RUNX2 is stably retained in the nucleus by its engagement with specific DNA and/or nucleoskeletal structures coexisting with the robustly condensed chromatin, in the MSCs biased toward an irreversible osteogenic differentiation.
\end{abstract}

Keywords : Mesenchymal stem cells, Osteogenic differentiation, Chromatin, Actin cytoskeleton, Histone deacetylase

\section{Introduction}

Mesenchymal stem cells (MSCs) have the potential for self-renewal and multipotency to differentiate into various lineages of tissues, including bone, cartilage, fat, and muscle (Pittenger et al., 1999). The MSCs can differentiate into different cell types under specific conditions in vitro and limited microenvironments in vivo, and the MSCs are, thus, of great interest in stem cell biology and in regenerative medicine as a component for tissue engineering (Pittenger et al., 2019). The lineage commitment with the loss of self-renewal and multilineage potential at early stages of MSC differentiation, in vitro, is determined sensitively by physical factors, such as stiffness (Engler et al., 2006), topography (Abagnale et al., 2015), and geometrical constraint (McBeath et al., 2004; Kilian et al., 2010) on the substrate, and cell density (Eyckmans et al., 2012), as well as the soluble chemical factors. Standard tissue culture polystyrene in vitro, biases the MSCs toward osteogenic differentiation irreversibly due to supraphysiological dose of the mechanical cue from the stiff surface (Yang et al., 2014). Elucidation of the mechanism for maintenance of self-renewal and multilineage potential in MSCs and the irreversible transition from MSCs into osteoblasts is a key research problem in basic stem cell 
biology.

In osteogenic differentiation, RUNX2 is known as a master transcriptional factor that regulates cell growth and phenotypic gene expression by integrated regulatory cues for physiological responsiveness (Zaidi et al., 2001). Numerous factors have been identified to be involved in the modulation of RUNX2 expression and transcriptional activity (Liu and Lee 2012). Among the upstream factors of RUNX2, yes-associated protein (YAP) is reported to be involved in the increase in Runx2 expression in response to the mechanical cues in the MSCs on stiff polystyrene surface (Yang et al., 2014).

Transcriptional regulator, YAP is a critical mediator that transduces the mechanical cues into various biological processes, via the regulation of the activities of transcription factors (Dupont et al., 2011; Kim et al., 2018; Wada et al., 2011). Intracellular localization of YAP depends on the actin organization. Actin development promotes nuclear localization of YAP (Dupont et al., 2011) and leads to enhanced expression of Runx2 in the MSCs (Yang et al., 2014). RUNX2 has nuclear localization signals (NLS) and is, thus, imported to the nucleus. In MSCs, the localization of RUNX2 can change reversibly from the nucleus to the cytoplasm in response to substrate softening through the suppression of the actin development (Yang et al., 2014). However, a long-term culture with conditions to retain YAP and RUNX2 in the nucleus induces loss of the reversibility, and RUNX2 is retained in nucleus even under conditions which suppress actin development (Yang et al., 2014). The mechanism of how RUNX2 is retained in the nucleus regardless of actin disturbance in osteogenic biased MSC has not been clarified.

A possible mechanism for the irreversible nuclear localization of RUNX2 is regulation through chromatin remodeling. Assembly and disassembly of chromatin regulated by histone modifications (Bannister and Kouzarides 2011) change accessibility of transcriptional factors to the DNA strands, resulting in changes to cellular gene expression (Martin and Cardoso 2010). It has been shown that an irreversible change in the chromatin structure occurs after longterm mechanical stimulation (Killaars et al., 2019). The mechanism of the nuclear retention of RUNX2 could be chromatin structure related, however it has not yet been completely clarified.

Here, to study the mechanism underlying the loss of lineage plasticity and robustness of irreversible osteogenic differentiation in the human MSCs, we investigated how RUNX2 was retained in the nucleus. To determine the contribution of the chromatin structure to the nuclear retention of RUNX2, we assayed the changes in the cellular localization of RUNX2 by perturbing chromatin structures in the human MSCs. Additionally, to unveil how actin cytoskeleton is involved in the signaling pathway, the link between the dynamic shuttling of RUNX2 and the actin organization, via YAP shuttling and chromatin condensation, was investigated.

\section{Materials and Methods}

\subsection{Cell culture}

Human bone marrow derived MSCs (American Type Culture Collection, Manassas, Virginia) were cultured in the mesenchymal stem cell growth medium (MSCGM, Lonza Japan, Tokyo, Japan) in a flask (Corning Japan, Tokyo, Japan) at $37{ }^{\circ} \mathrm{C}$ in a humidified atmosphere with $5 \% \mathrm{CO}_{2}$. After 4 passages, MSCs were detached and seeded onto a glasscoverslip bottomed 35-mm Petri dish (Matsunami Glass, Osaka, Japan) at $2000 \mathrm{cells} / \mathrm{cm}^{2}$. The cells were cultured in the MSCGM for 24 hours before the inhibitor treatment.

\subsection{Inhibitor treatment}

Actin polymerization inhibitor, latrunculin B (LatB, Abcam, Cambridge, UK) and a Rho kinase inhibitor, Y27632 (Focus Biomolecules, Plymouth Meeting, PA), were added to the MSCGM and used for the actin structure perturbations. The final concentrations were 80,400 , and $1000 \mathrm{nM}$ for Lat B, and 2.5, 10, and $25 \mu \mathrm{M}$ for Y27632. Histone deacetylase (HDAC) inhibitors, tricostatin A (TSA, FUJIFILM Wako Pure Chemical Corporation, Osaka, Japan) dissolved in DMSO and sodium butyrate ( $\mathrm{NaB}$, Sigma-Aldrich Japan, Tokyo, Japan) were added to the MSCGM and used for chromatin perturbations with chromatin decondensation. TSA is known to have side effects on cytoplasmic microtubules, whereas $\mathrm{NaB}$ has fewer side effects (Tran et al., 2007). The final concentrations were 10, 100, and $500 \mathrm{ng} / \mathrm{mL}$ for TSA, and 1, 10, and $50 \mathrm{mM}$ for $\mathrm{NaB}$. As a negative control, MSCs were cultured in MSCGM with $0.05 \%$ DMSO. The incubation time with each inhibitor was $0.5 \mathrm{~h}$, a time period shorter than the general turnover time of proteins, and 72 hours, which is longer than the general turnover time of proteins. 


\subsection{Fluorescent image acquisition}

MSCs were fixed with $4 \%$ paraformaldehyde (FUJIFILM Wako Pure Chemical Corporation) for 15 min, then permeabilized with $0.1 \%$ Triton X-100 (Sigma-Aldrich) for $5 \mathrm{~min}$, and blocked with $0.2 \%$ gelatin (Nacalai Tesque, Kyoto, Japan) in PBS $\left(\mathrm{Mg}^{2+}\right.$ and $\mathrm{Ca}^{2+}$ free) for $60 \mathrm{~min}$. The samples were incubated with primary antibodies, rabbit antiYAP (1:200, Cell Signaling Technology Japan, Tokyo, Japan) or rabbit anti-RUNX2 (1:1600, Cell Signaling Technology Japan) for YAP or RUNX2 labeling, respectively, at $4{ }^{\circ} \mathrm{C}$ for overnight. Subsequently, the cells were incubated with Alexa Fluor 555-labeled phalloidin (1:100, Cell Signaling Technology Japan), secondary antibody (1:1000, Cell Signaling Technology Japan) conjugated with Alexa Fluor 488, and DAPI ( $5 \mu \mathrm{g} / \mathrm{ml}$, Cell Signaling Technology Japan) for $60 \mathrm{~min}$ at room temperature.

The fluorescent images were acquired with a laser scanning confocal microscope (FV 3000, Olympus, Tokyo, Japan) with a UPlanSApo $60 \times \mathrm{NA}=1.42$ oil-immersion objective lens (Olympus). The z-slice images were acquired with the following settings: DAPI, Alexa Fluor 488, and Alexa Fluor 555 were excited with a $405 \mathrm{~nm}$ diode laser (Coherent Japan, Tokyo, Japan) of $1.0 \%$ intensity, a $488 \mathrm{~nm}$ diode laser (Coherent) of 1.0 - $3.0 \%$ intensity, and a 561 diode laser (Coherent) of $1.0 \%$ intensity, respectively; For all the fluorescent probes, pin hole diameter $=202 \mu \mathrm{m}$, detector sensitivity $=350$ $450 \mathrm{~V}$, scanning rate $=2.0 \mu \mathrm{s} /$ pixel, scanning size $=800 \times 800$ pixels at $0.265 \mu \mathrm{m}$ pixel pitch, and z-section interval $=$ $0.4 \mu \mathrm{m}$.

For the calculation of the nuclear to cytoplasmic ratios of YAP and that of RUNX2, a slice located in the nuclear thickness center was used. The ratios were obtained by dividing the mean fluorescent intensity of the nuclear area by mean fluorescent intensity of randomly selected five regions $(32 \times 32$ pixels at $0.265 \mu \mathrm{m}$ pixel pitch $)$ of the cytoplasm around the nucleus on the same slice. The nuclear region was determined from DAPI image.

\subsection{Statistical analysis}

Kolmogorov-Smirnov test was used to determine the normal distribution of the data. If data of all the groups to be compared were normally distributed, we used one-way ANOVA test as the parametric test and calculated the P-value between each group using Tukey's honestly significant difference test. If there was at least one group with data that were not normally distributed, we used Kruskal-Wallis test as the non-parametric test and calculated P-value using Bonferroni/Dunn test. A P-value less than 0.05 was considered statistically significant. Statistical comparisons were executed by Statistical Package for Social Science (SPSS).

\section{Results}

\subsection{Intranuclear localization of YAP and RUNX2 in MSCs cultured on the glass surface}

Figure 1 shows fluorescence images of the actin filaments, YAP, and RUNX2 in the MSCs cultured on glass surfaces for 24 hours just before the inhibitor treatments. As shown in the left panels of Fig. 1 (a) and (b), actin filaments were well developed. Both YAP and RUNX2 were localized in the nucleus, which was consistent with the previous report that YAP and RUNX2 localizes in the nucleus in MSCs cultured on a stiff substrate like polystyrene (Yang et al., 2014). The nuclear to cytoplasmic ratios of the fluorescent intensity were far above one for the diffusion state, as shown in Fig. 1(c). Inhibitor treatments were performed on these cells with YAP and RUNX2 in the nucleus.



(c)

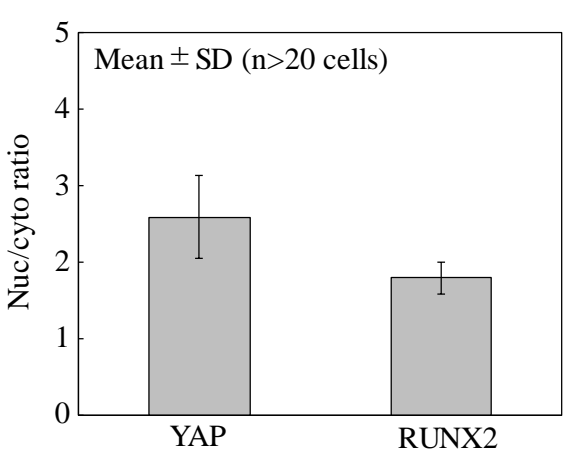

Fig. 1 Actin cytoskeletal organization and intracellular localization of YAP and RUNX2 just before the inhibitor treatment. (a) Fluorescence images of actin cytoskeleton (red), DNA (cyan), and (a) YAP and (b) RUNX2 (green). Scale bar, $50 \mu \mathrm{m}$. (c) Nuclear to cytoplasmic ratios of the fluorescence intensity of YAP and RUNX2. A representative result of three experiments is shown. 


\subsection{HDAC was necessary for nuclear localization of RUNX2 in human MSCs}

We first evaluated whether the organization of the actin cytoskeleton was related to the intranuclear localization of YAP and RUNX2, by using two different inhibitors disturbing the actin cytoskeletal integrity with different mechanisms of action. One of them was LatB, inhibiting actin polymerization. As shown in the leftmost panel of Fig. 2 (a) and (c), the LatB treated cells resulted in a decrease in the cell spreading area and the loss of actin fibers. In these cells, the YAP was diffused into the cytoplasm, whereas the RUNX2 remained in the nucleus. The result of the image analyses showed that the nuclear to cytoplasmic ratios of the fluorescent intensity were less than 1.0 for YAP (Fig. 2 (b) 1000 nM LatB) and more than 2.0 for RUNX2 (Fig. 2 (d) $1000 \mathrm{nM}$ LatB), confirming YAP diffusion and RUNX2 nuclear retention, respectively. Dose dependence analysis showed that the YAP diffusion and RUNX2 nuclear retention were evident with LatB concentrations ranging from $80 \mathrm{nM}$ to $1000 \mathrm{nM}$ (Fig. 2 (b) and (d)). The other inhibitor was Y27632 that suppresses myosin activity through inhibition of Rho kinase activity. As shown in the second column of Fig. 2 (a) and (c), in the Y27632 treated cells, cell spreading was suppressed and the number of thick actin fibers were decreased, but not as much as LatB treated cells. In these cells, the YAP was diffused into the cytoplasm, and the RUNX2 was remained in the nucleus with the nuclear to cytoplasmic ratios of the fluorescent intensity of less than 1.0 (Fig. 2 (b) $25 \mu \mathrm{M} \mathrm{Y27632)} \mathrm{and}$ more than 2.0 (Fig. 2 (d) $25 \mu \mathrm{M}$ Y27632), respectively. Dose-dependent analysis demonstrated that the effect of Y27632 on YAP diffusion increased in a dose dependent manner (Fig. 2 (b)), whereas that on the RUNX2 retention in the nucleus was almost constant, regardless of the concentration ranging from $2.5 \mu \mathrm{M}$ to $25 \mu \mathrm{M}$ (Fig. 2 (d)). These results suggested that YAP and RUNX2 did not always occur together in the nucleus. Thus, taken together, these results showed that the intracellular localization of YAP was determined depending on the magnitude of the actin organization, whereas RUNX2 retention was not actin dependent.

Next, we evaluated the possibility that chromatin organization was a determinant of the intranuclear localization of YAP and RUNX2, using TSA and NaB. Both TSA and NaB are known to cause chromatin decondensation through the inhibition of HDAC, but TSA is indicated to have a side effect on microtubules, whereas NaB does not (Tran et al., 2007). As shown in the third and fourth columns (from left) of Fig. 2 (a) and (c), cell spreading and actin organization were unaffected by both TSA and $\mathrm{NaB}$ treatments. YAP was retained in the nucleus (Fig. 2 (a)), with the nuclear to cytoplasmic ratios of fluorescent intensity above 2.0 for both TSA and NaB treatments (Fig. 2 (b) 500ng/mL TSA and $50 \mathrm{mM} \mathrm{NaB}$ ).
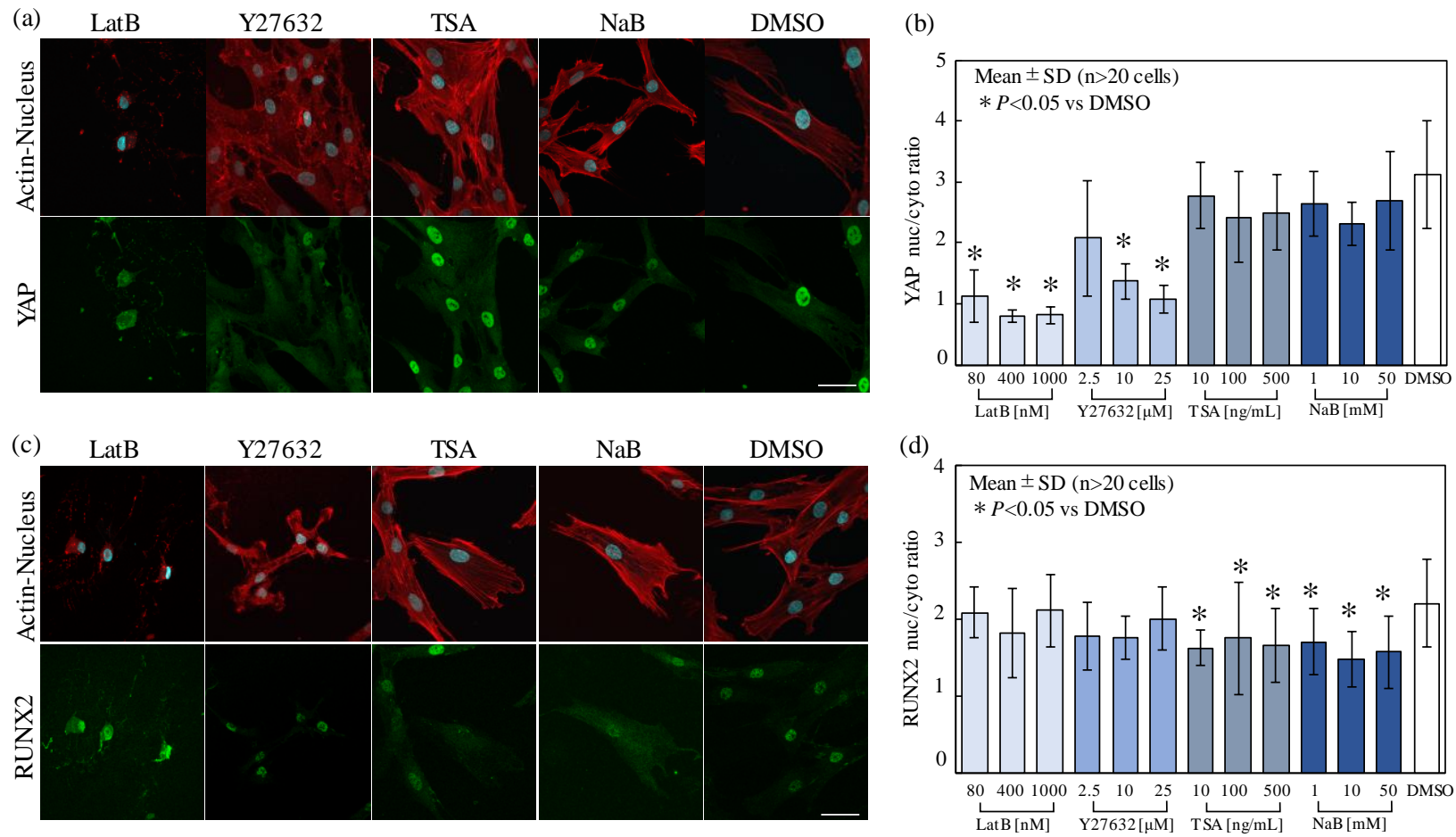

Fig. 2 Actin cytoskeletal organization and intranuclear localization of YAP and RUNX2 after $0.5 \mathrm{~h}$ incubation in the MSCGM with LatB, Y27632, TSA, NaB, and DMSO. (a, c) Fluorescence images of actin cytoskeleton (red), DNA (cyan), and (a) YAP and (c) RUNX2 (green) after $0.5 \mathrm{~h}$ incubation in the MSCGM with $1000 \mathrm{nM}$ LatB, $25 \mu \mathrm{M}$ Y27632, $500 \mathrm{ng} / \mathrm{mL}$ TSA, $50 \mathrm{mM} \mathrm{NaB}$, and DMSO. Scale bars, $50 \mu \mathrm{m}$. (b, d) Nuclear to cytoplasmic ratios of the fluorescence intensities of (b) YAP and (d) RUNX2 after the incubation. The results for the three experiments. 
As for RUNX2 shown in Fig. 2 (c), it remained in the nucleus; however, the magnitude of the accumulation seemed to be much lower with TSA and NaB treatments, compared to LatB and Y27632 treatments, and DMSO negative control. As shown in Fig. 2 (d), this observation was confirmed by the significant difference of the nuclear to cytoplasmic ratios of RUNX2 fluorescent intensity between the cells with TSA or NaB treatment and those with DMSO. RUNX2 diffusion by $\mathrm{NaB}$ as well as TSA treatments suggested that the RUNX2 localization was affected by HDAC inhibition itself or the resulting chromatin decondensation, but not by microtubule disturbance. In contrast with RUNX2, YAP localization was unaffected by the perturbation in the nuclear structure due to TSA and $\mathrm{NaB}$ treatments.

\subsection{The relationship between RUNX2 diffusion and nuclear morphology}

We finally investigated the nuclear changes that occurred with RUNX2 diffusion. As shown in Fig. 3 (a) and (b), the nucleus of the cells with the TSA treatments for $0.5 \mathrm{~h}$ expanded slightly compared to that of the cells before inhibitor treatment. After $24 \mathrm{~h}$ and $72 \mathrm{~h}$ inhibitor treatments, the nuclear expansion was maintained and RUNX2 was diffused from

(a)

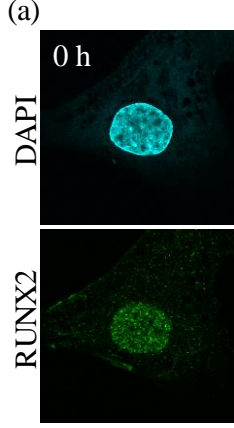

(b)

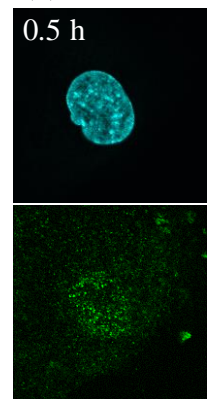

(c)



(d)

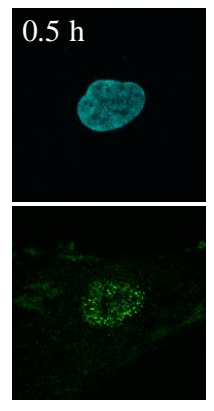

TSA $500 \mathrm{ng} / \mathrm{mL}$



$\mathrm{NaB} 50 \mathrm{mM}$


DMSO

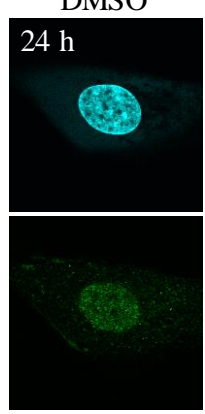

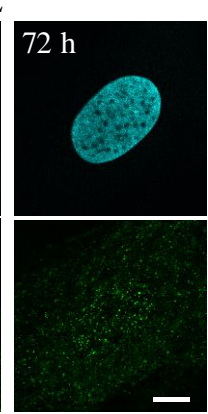
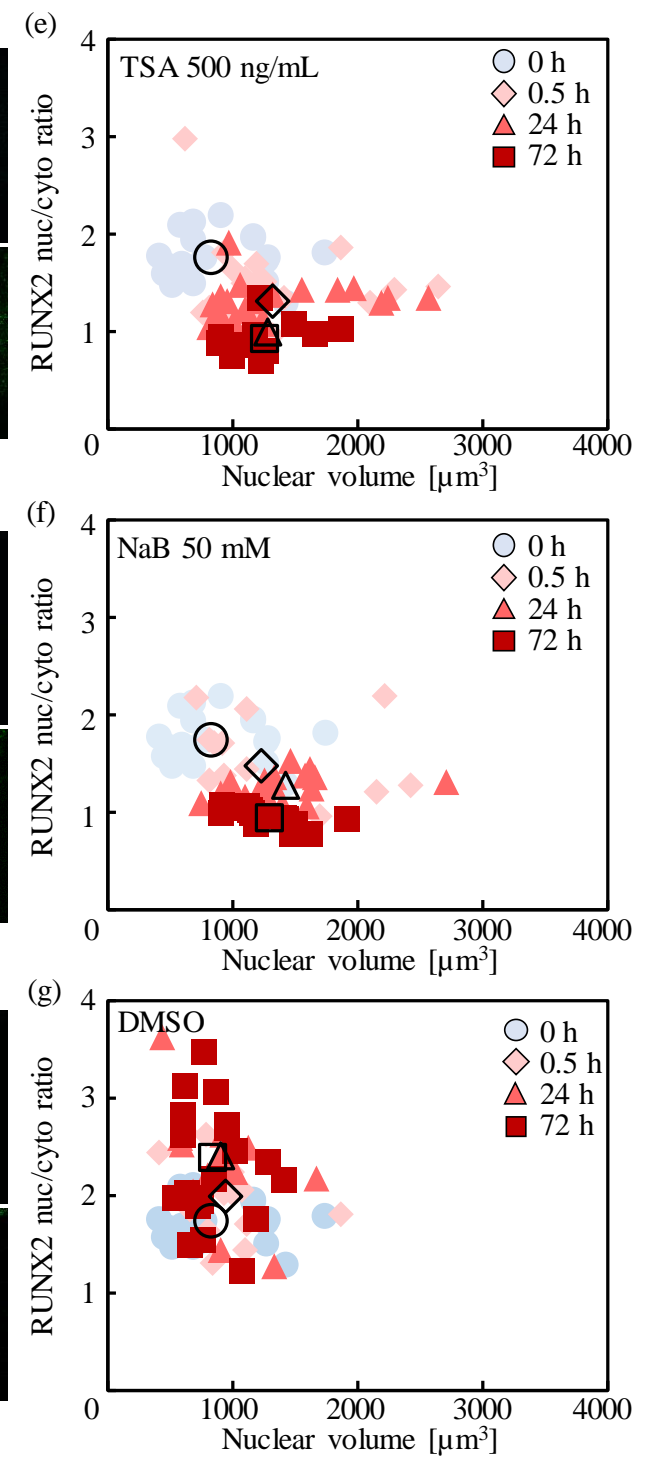
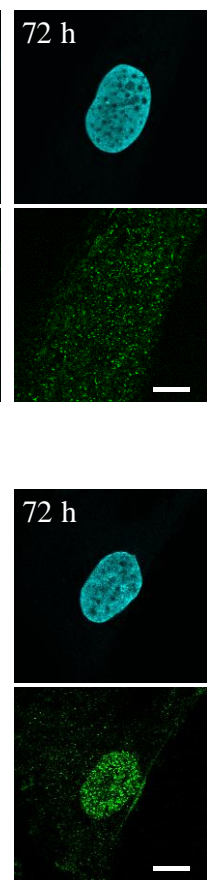

Fig. 3 Nuclear morphology and intranuclear localization of RUNX2. (a-d) Fluorescence images of DNA (cyan) and RUNX2 (green) (a) before, and (b-d) after $0.5 \mathrm{~h}, 24 \mathrm{~h}$, and $72 \mathrm{~h}$ incubations in the MSCGM with (b) $500 \mathrm{ng} / \mathrm{mL}$ TSA, (c) $50 \mathrm{mM} \mathrm{NaB}$, and (d) DMSO. Scale bars, $10 \mu \mathrm{m}$. (e-g) Scatter plots of cell nuclear volume (x-axis) versus nuclear to cytoplasmic ratios of RUNX2 (y-axis) after $0.5 \mathrm{~h}, 24 \mathrm{~h}$, and $72 \mathrm{~h}$ incubations in the MSCGM with (e) $500 \mathrm{ng} / \mathrm{mL}$ TSA, (f) $50 \mathrm{mM} \mathrm{NaB}$, and (g) DMSO. The black circle, rhombus, triangle, and rectangle show the mean values of cell nuclear volume and the nuclear to cytoplasmic ratio of RUNX2 before, and after $0.5 \mathrm{~h}, 24 \mathrm{~h}$, and $72 \mathrm{~h}$ incubations, respectively. Each plot is a representative result of the three experiments. 
the nucleus to the cytoplasm. These tendencies were also observed in the cells with NaB treatment (Fig. 3 (c)). The nuclear expansion and RUNX2 diffusion were not observed in the cells with DMSO treatment as a negative control (Fig. 3 (d)). Nuclear expansion is supposed to be caused due to the chromatin decondensation by HDAC inhibitors (Rao et al., 2007). The result of the quantitative analysis in Fig. 3 (e), (f), and (g) showed that the nuclear expansion in the cells with the TSA and $\mathrm{NaB}$ treatments was detected as larger values of the volume of the nucleus relative to the DMSO negative control. In addition, after $0.5 \mathrm{~h}$ TSA and $\mathrm{NaB}$ treatments, nuclear volumes were increased and nuclear to cytoplasmic ratios of the fluorescent intensity of RUNX2 were slightly decreased to approximately 1.5 , indicating that nuclear expansion was occurred even when RUNX2 were localized in nucleus. Then, after $24 \mathrm{~h}$ or $72 \mathrm{~h}$, although nuclear volumes were equivalent to that of the cells with the inhibitor treatment for $0.5 \mathrm{~h}$, the nuclear to cytoplasmic ratios of RUNX2 were decreased to 1.0. In the DMSO negative control, the increase in nuclear volume and the decrease in nuclear to cytoplasmic ratios of RUNX2 were not occurred (Fig. 3 (g)). In contrast to TSA and NaB, LatB and Y27632 treatments caused nuclear contraction without RUNX2 translocation (Fig. 4 (a) and (b)). Temporal changes in the nuclear morphology and RUNX2 localization in the inhibitor assays demonstrated that the RUNX2 diffusion from the nucleus was promoted with nuclear expansion that was probably caused by chromatin decondensation.


Fig. 4 Nuclear morphology and intranuclear localization of RUNX2 after actin disturbance. (a) The fluorescence images after $0.5 \mathrm{~h}$ incubation in the MSCGM with $1000 \mathrm{nM}$ LatB, 25 $\mu$ M Y27632, and DMSO. Scale bar, $10 \mu \mathrm{m}$. (b) Scatter plot of nuclear volume (x-axis) versus nuclear to cytoplasmic ratios of RUNX2 (y-axis) after $0.5 \mathrm{~h}$ incubation. The black circle, rectangle, and cross show the mean values of nuclear volume and the nuclear to cytoplasmic ratio of RUNX2 for $1000 \mathrm{nM}$ LatB, $25 \mu \mathrm{M}$ Y27632, and DMSO, respectively. The result for more than 20 cells in one experiment is shown.

\section{Discussion}

Collectively, our results showed that condensed chromatin structure was necessary for localization of RUNX2 to the nucleus. The nuclear retention of RUNX2 was unaffected due to the disruption of actin cytoskeleton by inhibition of actin polymerization and that of actomyosin force generation under the condition that chromatin condensation was maintained, although YAP was translocated from the nucleus to the cytoplasm.

Human MSCs cultured on stiff matrices with elastic moduli that mimic collagenous bone or higher tend to undergo osteogenic differentiation (Engler et al., 2006; Yang et al., 2014). In osteogenic lineage cells, RUNX2 is a master regulator (Zaidi et al., 2001), and YAP is selectively expressed and serves as a co-regulator for the transcription factors, including RUNX2 (Pan et al., 2018). We showed that YAP and RUNX2 were localized to the nucleus in the MSCs during the expansion culture with four passages on the polystyrene surfaces, followed by 24 hours culture on the glass surface, just before the inhibitor treatments (Fig. 1). The nuclear accumulations of YAP and RUNX2 suggested that the MSCs just before the inhibitor treatments were biased into the osteogenic lineage due to the mechanical dosing during the preculture.

Numerous studies have shown that YAP shuttling between the nucleus and the cytoplasm is affected by the organization of the actin cytoskeleton. Thick actin bundles and actin cytoskeletal tension suppresses various proteins activity, such as Lats1/2 in Hippo pathway, and angiomotin that induces YAP diffusion from the nucleus into cytoplasm and YAP cytoplasmic retention (Brusatin et al., 2018). Similarly, YAP was diffused into the cytoplasm by 
the inhibition of actin polymerization with LatB and that of Rho/ROCK signaling pathway with Y27632 (Fig. 2 (a) and (b)).

Interestingly, while our data was consistent for YAP localization, regulation of RUNX2 localization seemed to be more complex. Runx2 expression is activated by YAP translocated to the nucleus in the MSCs (Yang et al., 2014) and RUNX2 is assumed to be lost from the nucleus due to the suppression of its gene expression through YAP diffusion into the cytoplasm by LatB and Y27632 treatments in a culture period longer than the RUNX2 turnover time. However, on a shorter time scale, this study showed that RUNX2 was retained in the nucleus by the LatB and Y27632 treatments (Fig. 2 (c) and (d)).

One possible mechanism to explain the RUNX2 dynamics is modulation of RUNX2 affinity to DNA and/or nucleoskeleton, which is affected by the chromatin condensation state. Inhibition of HDAC resulted in diffusion of RUNX2 from the nucleus into the cytoplasm (Fig. 2 (c) and (d)) and nuclear expansion (Fig. 3 (e) and (f)). Our analysis further demonstrated that the RUNX2 diffusion from the nucleus occurred later in time than the nuclear expansion (Fig. 3 (e) and (f)). Nuclear expansion is reported to be accompanied by chromatin decondensation in the cells treated by HDAC inhibitors (Toth et al., 2004; Rao et al., 2007). Chromatin organization affects the interaction of transcription factors with DNA, and thus, the gene expression (Martin and Cardoso 2010; Voss and Hager 2014). RUNX2 is known as a transcription factor which has a binding domain to nucleoskeleton (Zaidi et al., 2001; Quirós-González et al., 2016) as well as DNA (Thirunavukkarasu et al., 1998). In our assay, the decrease in nuclear RUNX2 started to occur in $0.5 \mathrm{~h}$ of incubation (Fig. 2 (c) and (d), Fig. 3 (b) and (c)), which was much shorter than the generally known half-life of the protein (Shamir et al., 2016). Therefore, RUNX2 diffusion will not occur due to RUNX2 depletion, but due to its translocation from the nucleus to the cytoplasm owing to loss of affinity of RUNX2 to DNA and/or nucleoskeleton. Based on these findings, modulation of the affinity could be caused by the nuclear expansion with chromatin decondensation caused by HDAC inhibition.

In contrast to the HDAC-inhibited cells, both in the cells with inhibited actin polymerization and in those with inhibited ROCK, RUNX2 was retained in the nucleus and the cell nucleus was not expanded but contracted (Fig. 4 (a) and (b)). In these cells, chromatin organization will be either unaffected or condensed. RUNX family proteins might have the potential to engage condensed chromatin to facilitate its remodeling (Mevel et al., 2019). It might be also the case for RUNX2 retention in the regulation of osteogenic differentiation in the human MSCs.

In human MSCs, reversibility of chromatin organization is shown to be dependent on the culture history. Specifically, long-term mechanical dosing with a stiff (Young's modulus, around $30 \mathrm{kPa}$ ) substrate induces irreversible chromatin organization (Killaars et al., 2019). The irreversible chromatin condensation will lead to persistent RUNX2 retention in the nucleus, which result in depletion of multilineage potential, and alternatively robust and irreversible progression of osteogenic differentiation.

In conclusion, our experiments revealed that condensed chromatin was associated with nuclear retention of RUNX2 in osteogenic differentiation-biased hMSCs. These insights contribute to clarify the mechanism underlying the plasticity of human MSCs and the irreversible osteogenic differentiation of MSCs through modulation of the interaction of RUNX2 with DNA and/or nucleoskeleton depending on the chromatin condensation state. Recent studies have shown that actin cytoskeletal tension induces chromatin remodeling ( $\mathrm{Li}$ et al., 2014) and that local stress applied to integrin propagate the tensed actin cytoskeleton to the intranuclear region to stretch the chromatin (Tajik et al., 2016). Based on these findings, a mechanical cue to modulate the actin cytoskeletal dynamics that causes chromatin decondensation could prevent RUNX2 from persistent nuclear localization, which enables the maintenance of pluripotency of MSCs. In the future, we will elucidate the mechanism underlying actin cytoskeletal dynamics that leads to chromatin remodeling, which affects the cellular localization of RUNX2 and other critical transcription factors. The findings can form a basis to build a successful strategy to maintain plasticity of MSCs and robust osteogenic differentiation.

\section{Acknowledgments}

Author contributions: M.Y., H.F., and H.M. developed the concept of the study. M.Y. performed majority of the experiments and analyzed the data. M.Y. and H.M. wrote the manuscript. We thank Naoya Sakamoto for helpful advice and comments. 


\section{Funding}

This work was supported by JSPS KAKENHI Grant Number JP19J13048 and AMED under Grant Number 18 gm5810012h9904.

\section{Conflicts of interest}

The authors declare no competing financial interests.

\section{References}

Abagnale, G., Steger, M., Nguyen, V. H., Hersch, N., Sechi, A., Joussen, S., Denecke, B., Merkel, R., Hoffmann, B., Dreser, A., Schnakenberg, U., Gillner, A. and Wagner, W., Surface topography enhances differentiation of mesenchymal stem cells towards osteogenic and adipogenic lineages, Biomaterials, Vol.61, (2015), pp.316-326.

Bannister, A. J. and Kouzarides, T., Regulation of chromatin by histone modifications, Cell Res, Vol.21, No.3 (2011), pp.381-395.

Brusatin, G., Panciera, T., Gandin, A., Citron, A. and Piccolo, S., Biomaterials and engineered microenvironments to control YAP/TAZ-dependent cell behaviour, Nat Mater, Vol.17, No.12 (2018), pp.1063-1075.

Dupont, S., Morsut, L., Aragona, M., Enzo, E., Giulitti, S., Cordenonsi, M., Zanconato, F., Le Digabel, J., Forcato, M., Bicciato, S., Elvassore, N. and Piccolo, S., Role of YAP/TAZ in mechanotransduction, Nature, Vol.474, No.7350 (2011), pp.179-183.

Engler, A. J., Sen, S., Sweeney, H. L. and Discher, D. E., Matrix elasticity directs stem cell lineage specification, Cell, Vol.126, No.4 (2006), pp.677-689.

Eyckmans, J., Lin, G. L. and Chen, C. S., Adhesive and mechanical regulation of mesenchymal stem cell differentiation in human bone marrow and periosteum-derived progenitor cells, Biol Open, Vol.1, No.11 (2012), pp.1058-1068.

Kilian, K. A., Bugarija, B., Lahn, B. T. and Mrksich, M., Geometric cues for directing the differentiation of mesenchymal stem cells, Proc Natl Acad Sci U S A, Vol.107, No.11 (2010), pp.4872-4877.

Killaars, A. R., Grim, J. C., Walker, C. J., Hushka, E. A., Brown, T. E. and Anseth, K. S., Extended Exposure to Stiff Microenvironments Leads to Persistent Chromatin Remodeling in Human Mesenchymal Stem Cells, Adv Sci (Weinh), Vol.6, No.3 (2019), DOI:10.1002/advs.201801483.

Kim, M. K., Jang, J. W. and Bae, S. C., DNA binding partners of YAP/TAZ, BMB Rep, Vol.51, No.3 (2018), pp.126-133.

Li, Q., Kumar, A., Makhija, E. and Shivashankar, G. V., The regulation of dynamic mechanical coupling between actin cytoskeleton and nucleus by matrix geometry, Biomaterials, Vol.35, No.3 (2014), pp.961-969.

Liu, T. M. and Lee, E. H., Transcriptional Regulatory Cascades in Runx2-Dependent Bone Development, Tissue Engineering Part B: Reviews, Vol.19, No.3 (2012), pp.254-263.

Martin, R. M. and Cardoso, M. C., Chromatin condensation modulates access and binding of nuclear proteins, FASEB J, Vol.24, No.4 (2010), pp.1066-1072.

McBeath, R., Pirone, D. M., Nelson, C. M., Bhadriraju, K. and Chen, C. S., Cell shape, cytoskeletal tension, and RhoA regulate stem cell lineage commitment, Dev Cell, Vol.6, No.4 (2004), pp.483-495.

Mevel, R., Draper, J. E., Lie-a-Ling, M., Kouskoff, V. and Lacaud, G., RUNX transcription factors: orchestrators of development, Development, Vol.146, (2019), dev148296.

Pan, J. X., Xiong, L., Zhao, K., Zeng, P., Wang, B., Tang, F. L., Sun, D., Guo, H. H., Yang, X., Cui, S., Xia, W. F., Mei, L. and Xiong, W. C., YAP promotes osteogenesis and suppresses adipogenic differentiation by regulating betacatenin signaling, Bone Res, Vol.6, No.18 (2018). DOI: 10.1038/s41413-018-0018-7

Pittenger, M. F., Discher, D. E., Péault, B. M., Phinney, D. G., Hare, J. M. and Caplan, A. I., Mesenchymal stem cell perspective: cell biology to clinical progress, NPJ Regen Med, Vol.4, No.1 (2019). DOI: 10.1038/s41536-019-00836

Pittenger, M. F., Mackay, A. M., Beck, S. C., Jaiswal, R. K., Douglas, R., Mosca, J. D., Moorman, M. A., Simonetti, D. W., Craig, S. and Marshak, D. R., Multilineage potential of adult human mesenchymal stem cells, Science, Vol.284, (1999), pp.143-147.

Quirós-González, I., Román-García, P., Alonso-Montes, C., Barrio-Vázquez, S., Carrillo-López, N., Naves-Díaz, M., Mora, M. I., Corrales, F. J., López-Hernández, F. J., Ruiz-Torres, M. P., Cannata-Andía, J. B. and Fernández-Martín, J. L., Lamin A is involved in the development of vascular calcification induced by chronic kidney failure and 
phosphorus load, Bone, Vol.84, (2016), pp.160-168.

Rao, J., Bhattacharya, D., Banerjee, B., Sarin, A. and Shivashankar, G. V., Trichostatin-A induces differential changes in histone protein dynamics and expression in HeLa cells, Biochemical and Biophysical Research Communications, Vol.363, No.2 (2007), pp.263-268.

Shamir, M., Bar-On, Y., Phillips, R. and Milo, R., SnapShot: Timescales in cell biology, Cell, Vol.164, No.6 (2016), pp.1302-1302.

Tajik, A., Zhang, Y., Wei, F., Sun, J., Jia, Q., Zhou, W., Singh, R., Khanna, N., Belmont, A. S. and Wang, N., Transcription upregulation via force-induced direct stretching of chromatin, Nature Materials, Vol.15, No.12 (2016), pp.12871296.

Thirunavukkarasu, K., Mahajan, M., McLarren, K.W., Stifani, S. and Karsenty, G., Two domains unique to osteoblastspecific transcription factor Osf $2 / \mathrm{Cbfa} 1$ contribute to its transactivation function and its inability to heterodimerize with Cbfbeta, Mol Cell Biol1, Vol.18, No.7 (1998), pp.4197-4208.

Toth, K. F., Knoch, T. A., Wachsmuth, M., Frank-Stohr, M., Stohr, M., Bacher, C. P., Muller, G. and Rippe, K., Trichostatin A-induced histone acetylation causes decondensation of interphase chromatin, J Cell Sci, Vol.117, (2004), pp.4277-4287.

Tran, A. D.-A., Marmo, T. P., Salam, A. A., Che, S., Finkelstein, E., Kabarriti, R., Xenias, H. S., Mazitschek, R., Hubbert, C., Kawaguchi, Y., Sheetz, M. P., Yao, T.-P. and Bulinski, J. C., HDAC6 deacetylation of tubulin modulates dynamics of cellular adhesions, J Cell Sci, Vol.120, (2007), pp.1469-1479.

Voss, T. C. and Hager, G. L., Dynamic regulation of transcriptional states by chromatin and transcription factors, Nature Reviews Genetics, Vol.15, No.2 (2014), pp.69-81.

Wada, K., Itoga, K., Okano, T., Yonemura, S. and Sasaki, H., Hippo pathway regulation by cell morphology and stress fibers, Development, Vol.138, No.18 (2011), pp.3907-3914.

Yang, C., Tibbitt, M. W., Basta, L. and Anseth, K. S., Mechanical memory and dosing influence stem cell fate, Nat Mater, Vol.13, No.6 (2014), pp.645-652.

Zaidi, S. K., Javed, A., Choi, J. Y., van Wijnen, A. J., Stein, J. L., Lian, J. B. and Stein, G. S., A specific targeting signal directs Runx2/Cbfa1 to subnuclear domains and contributes to transactivation of the osteocalcin gene, J Cell Sci, Vol.114, No.17 (2001), pp.3093-3102. 